\title{
Chapter 37 \\ Meta-analysis of Resistance to Fusarium Head Blight in Tetraploid Wheat: Implications for Durum Wheat Breeding
}

\author{
Noémie Prat, Maria Buerstmayr, Barbara Steiner, and Hermann Buerstmayr
}

\begin{abstract}
Improvement of resistance to Fusarium head blight (FHB) is a continuous challenge for durum wheat (Triticum durum) breeding, where most germplasm are susceptible and low genetic variation is available for this trait. Research has focused on broadening the genetic basis by introducing alleles for FHB resistance from landraces and related species such as bread wheat (Triticum aestivum), cultivated emmer (Triticum dicoccum), wild emmer (Triticum dicoccoides) and Persian wheat (Triticum carthlicum) into durum wheat. We summarize and compare here QTL mapping studies carried out to date in tetraploid wheat. Thirteen QTL with small to moderate effects were repeatedly detected on 11 chromosomes with alleles improving FHB resistance deriving from relatives and from durum wheat itself. Comparison showed large overlaps of QTL positions with those identified in hexaploid wheat suggesting a common genetic basis for FHB resistance. FHB resistance breeding by allele introgression into durum wheat is feasible and QTL pyramiding in novel cultivars is a promising strategy for resistance breeding.
\end{abstract}

Keywords Durum wheat $\bullet$ Fusarium head blight $\bullet$ QTL $\bullet$ Resistance $\bullet$ Tetraploid wheat $•$ Triticum durum

\footnotetext{
N. Prat

Department for Agrobiotechnology, BOKU-University of Natural Resources and Life Sciences, Vienna, Konrad Lorenz Str. 20, Tulln 3430, Austria

Florimond-Desprez, 3 rue Florimond-Desprez, BP 41, Cappelle-en-Pévèle 59242, France

INRA-Université Blaise Pascal, Genetic, Diversity and Ecophysiology of Cereals, UMR 1095, 5 chemin de Beaulieu, Clermont-Ferrand Cedex 2 63039, France

M. Buerstmayr $(\bowtie) \bullet B$. Steiner $\bullet$ H. Buerstmayr

Department for Agrobiotechnology, BOKU-University of Natural Resources and Life

Sciences, Vienna, Konrad Lorenz Str. 20, Tulln 3430, Austria

e-mail: maria.buerstmayr@boku.ac.at
} 


\section{Introduction}

Durum wheat (Triticum durum) is the principal cultivated tetraploid wheat species. Its annual production accounts for $\sim 5 \%$ of the total wheat grown worldwide and it is used mainly for preparation of pasta and semolina (Taylor and Koo 2012). Durum wheat, as other small grain cereals, suffers from susceptibility to Fusarium head blight (FHB), a devastating disease that affects wheat growing regions throughout the world. FHB is caused by a broad range of fungi from the Fusarium genera (Xu and Nicholson 2009). FHB epidemics are a serious threat for wheat production as the disease leads not only to yield losses but also infests crops with potent mycotoxins hazardous for food safety (Pestka 2010). This is particularly alarming in durum wheat since it is predominantly intended for direct human consumption.

An increasing demand for pasta products has led to an expansion of durum wheat production zones from traditional warm and dry cropping areas to more humid regions with climatic conditions conducive to the disease. Solutions to prevent FHB damages are limited and the development of resistant cultivars is considered a sustainable and highly desired approach to reduce FHB damages (Bai and Shaner 2004).

\section{FHB Resistance in Durum Wheat}

Current durum cultivars are generally susceptible to FHB (Clarke et al. 2010; authors' unpublished results). Sources of resistance remain scarce despite efforts undertaken to discover FHB resistant lines: large collections of thousands of durum wheat accessions have been screened without identifying resistant lines (Elias et al. 2005). Surveys on material from CIMMYT and ICARDA identified only five lines from a Tunisian source with moderate resistance to FHB spread (Huhn et al. 2012) and four Syrian landraces with stable resistance (Talas et al. 2011). The lack of resistance found in durum wheat may be attributed to historically low exposure to FHB and to the limited breeding efforts put into this relatively modern crop, which led to a narrow genetic base compared to other wheat species (Ban et al. 2005; Oliver et al. 2008). It is also speculated that durum carries susceptibility factors and/ or suppressor genes that compromise FHB resistance (Stack et al. 2002; Garvin et al. 2009; Ghavami et al. 2011).

Studies have thus been directed at evaluating relatives of durum wheat in order to broaden the genetic basis for breeding and efforts targeted at transferring FHB resistance into durum wheat.

In hexaploid wheat (Triticum aestivum) more than 100 QTL for FHB resistance have been mapped and some have been successfully integrated in breeding programs through marker assisted selection (Buerstmayr et al. 2009). Yet, attempts to transfer resistance into tetraploid wheat have met limited success (Oliver et al. 2007; authors' unpublished results). One hypothetical explanation for the often 
disappointingly low effect of hexaploid wheat QTL alleles when transferred into durum wheat is that the D-genome, absent in tetraploid wheat, contributes resistanceinducing factors (Fakhfakh et al. 2011).

Consequently resistance has been sought in tetraploid close relatives of durum wheat, where transfer of resistance is not confounded by differences in ploidy levels. Moderate to good FHB resistant tetraploid accessions have been successfully identified (Oliver et al. 2008; Buerstmayr et al. 2003).

\section{QTL Studies in Tetraploid Wheat}

QTL mapping studies carried out to date in durum wheat have been based on resistance deriving from tetraploid sources including wild emmer Triticum dicoccoides, cultivated emmer Triticum dicoccum, Persian wheat Triticum carthlicum and durum wheat landraces. A list including information on the resistance source used in the mapping analysis, the inoculation methods performed and the type of resistance assessed for each study is given in Table 37.1.

We gather here QTL reported in tetraploid wheat which were repeatedly found in different years or in independent studies. A total of 13 small to moderately effective QTL were mapped to 11 chromosomes. Their positions are indicated with vertical bars in Fig. 37.1, and the names of the genotypes contributing to resistance allele and the applied inoculation methods are also specified.

Durum wheat itself contributed resistance-improving alleles for the QTL on 2B (Gladysz et al. 2007; Somers et al. 2006), 3B (Buerstmayr et al. 2012; Ghavami et al. 2011) and 5B (Ghavami et al. 2011). This backs up the idea that in current durum wheat a certain level of FHB resistance is already available. A potential susceptibility factor which increases durum wheat susceptibility was detected on $2 \mathrm{~A}$

Table 37.1 QTL studies carried out in durum wheat mentioning resistance source, inoculation method and type of resistance evaluated

\begin{tabular}{l|l|l|l|l}
\hline Resistance source & Inoc. & Resistance & \\
\hline T. dicoccoides & Israel A (2A; 3A) & SFI & FHB spread & $\begin{array}{l}\text { Otto et al. (2002); Chen et al. } \\
\text { (2007); Garvin et al. (2009) }\end{array}$ \\
\hline T. dicoccoides & PI478742 (7A) & SFI & FHB spread & Kumar et al. (2007) \\
\hline T. dicoccoides & Mt.Hermon\#22 & SFI & FHB spread & Gladysz et al. (2007) \\
\hline T. dicoccoides & Mt.Gerizim\#36 & SFI & FHB spread & Buerstmayr et al. (2013) \\
\hline$T$. carthlicum & Blackbird & SFI & FHB spread & Somers et al. (2006) \\
\hline T. dicoccum & T. dic-161 & spray & FHB severity & Buerstmayr et al. (2012) \\
\hline T. dicoccum & BGRC3487 & SFI & FHB spread & Ruan et al. (2012) \\
\cline { 2 - 4 } & & spray & FHB severity & \\
\hline T. durum & 4 Tunisian lines & SFI & FHB spread & Ghavami et al. (2011) \\
\hline
\end{tabular}

SFI single floret inoculation, spray spray inoculation, FHB spread resistance to spread of the disease within the spike (type 2 resistance), FHB severity disease severity per plot after spray inoculation 

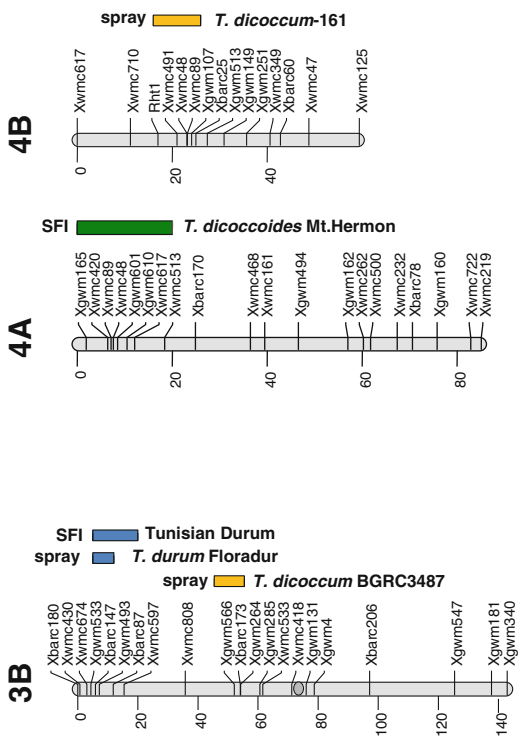
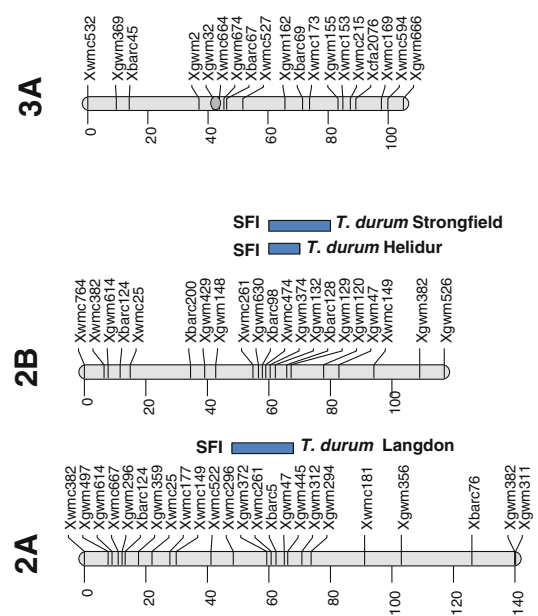

SFI $\square$. durum Strongfield SFI $\square T$. durum Helidur
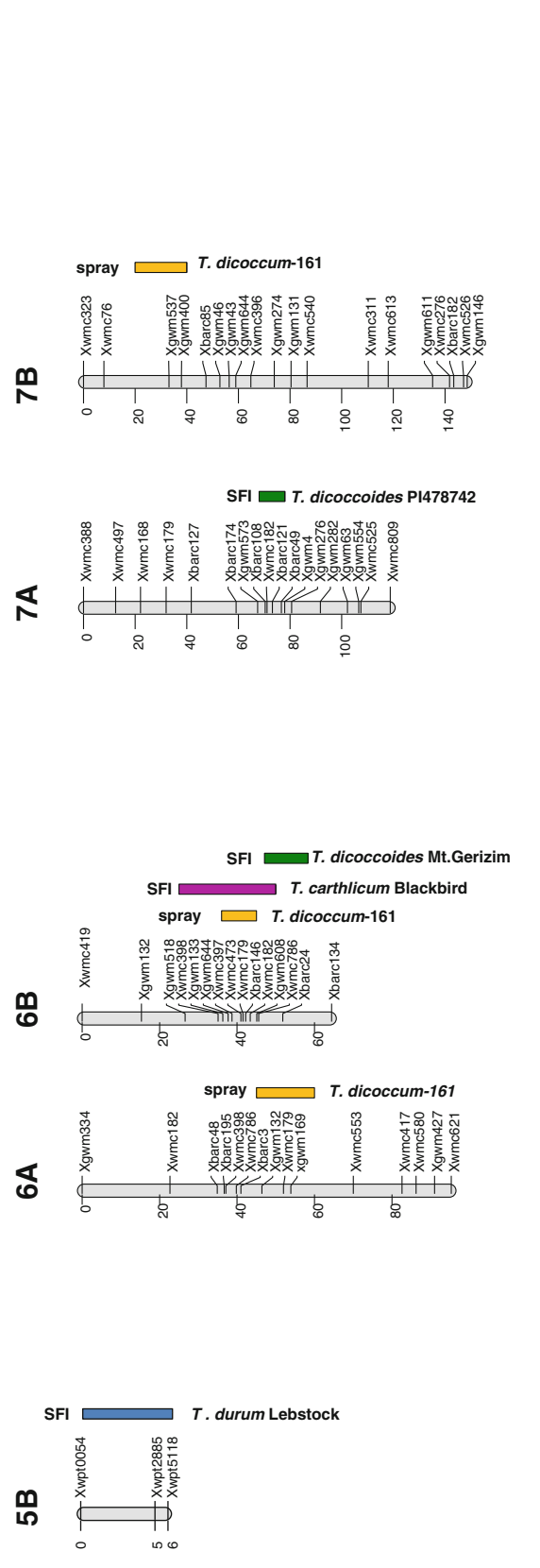
derived from T. dicoccoides Israel A (Garvin et al. 2009; Stack et al. 2002). Ghavami et al. (2011) also suspected the existence of a QTL influencing FHB resistance in the same chromosomal region in durum wheat.

Positions of many of the resistance QTL identified in tetraploid wheat coincided with QTL discovered in hexaploid wheat, suggesting common genes for resistance: e.g. QTL on 2B, 3A, 3B, 6B and 7B were found in the same regions where several QTL have been reported in hexaploid wheat (Buerstmayr et al. 2009). Positions of the QTL on 3B and 6B overlapped with those of the well documented genes Fhb1 and $F h b 2$, respectively, which were first described in the hexaploid cultivar Sumai-3 (Buerstmayr et al. 2009). Allele survey at these loci by Buerstmayr et al. (2012) revealed different SSR marker haplotypes between tetraploid lines and Sumai-3. The existence of resistance improving alleles at these loci in tetraploid wheat may circumvent the need to transfer resistance from hexaploid Asian sources into durum wheat.

Developmental and morphological traits often correlate with FHB response both in hexaploid wheat (Buerstmayr et al. 2009) and in tetraploid wheat. For example, under field conditions with spray inoculation a large effect QTL for FHB resistance was mapped at the position of the major plant height gene Rht-Bl on chromosome $4 \mathrm{~B}$ and a FHB resistance QTL on 7B coincided with a QTL for heading date (Buerstmayr et al. 2012). It is not clear yet whether or not these genes have pleiotropic effects or rather an indirect influence on FHB resistance due to plant height and flowering date per se.

\section{Conclusions and Perspectives}

Only few accessions have been used as sources for FHB resistance in durum wheat to date, yet results are promising, yielding multiple QTL with small to medium effects. Common genetic basis for FHB resistance in tetraploid and hexaploid wheat is likely as the positions of their QTL overlap to a large extent. Introgression of positive alleles into durum wheat is feasible and markers located near the mapped QTL are amenable for marker-assisting backcrossing. Pyramiding multiple resistance improving QTL combined with selection against suspected susceptibility factors is a promising breeding strategy to improve FHB resistance in novel cultivars. Improvements in durum wheat breeding are underway. Recently, the evaluation of novel experimental lines descending from multiple crosses of T. durum with T. aestivum, T. dicoccum and T. dicoccoides in our field trials in Tulln (Austria) showed enhanced variation for FHB resistance including lines with improved and stable FHB resistance performance.

Acknowledgments We gratefully acknowledge financial support from the Austrian Science Fund (FWF), projects P17310-B05 and F3711, and from the French Ministry of Higher Education and Research, CIFRE funding 2012/1405. 
Open Access This chapter is distributed under the terms of the Creative Commons Attribution Noncommercial License, which permits any noncommercial use, distribution, and reproduction in any medium, provided the original author(s) and source are credited.

\section{References}

Bai G, Shaner G (2004) Management and resistance in wheat and barley to Fusarium head blight. Annu Rev Phytopathol 42:135-161

Ban T, Kishii M, Ammar K et al (2005) CIMMYT's challenges for global communication and germplasm enhancement for FHB resistance. In: Canty S, Lewis J, Siler L, Ward R (eds) Proceedings of national Fusarium head blight forum. East Lansing. Michigan State University, Milwaukee, pp 6-10

Buerstmayr H, Stierschneider M, Steiner B, Lemmens M, Griesser M, Nevo E, Fahima T (2003) Variation for resistance to head blight caused by Fusarium graminearum in wild emmer (Triticum dicoccoides) originating from Israel. Euphytica 130:17-23

Buerstmayr H, Ban T, Anderson J (2009) QTL mapping and marker assisted selection for Fusarium head blight resistance in wheat: a review. Plant Breed 128:1-26

Buerstmayr M, Huber K, Heckmann J et al (2012) Mapping of QTL for Fusarium head blight resistance and morphological and developmental traits in three backcross populations derived from Triticum dicoccum $\times$ Triticum durum. Theor Appl Genet 125:1751-1765

Buerstmayr M, Alimari A, Steiner B, Buerstmayr H (2013) Genetic mapping of QTL for resistance to Fusarium head blight spread (type 2 resistance) in a Triticum dicoccoides $\times$ Triticum durum backcross-derived population. Theor Appl Genet 126:2825-2834

Chen X, Faris J, Hu J et al (2007) Saturation and comparative mapping of a major Fusarium head blight resistance QTL in tetraploid wheat. Mol Breed 19:113-124

Clarke J, Clarke F, Pozniak C (2010) Forty-six years of genetic improvement in Canadian durum wheat cultivars. Can J Plant Sci 90:791-801

Elias E, Manthey F, Stack R, Kianian S (2005) Breeding efforts to develop Fusarium head blight resistant durum wheat in North Dakota. In: Canty S, Lewis J, Siler L, Ward R (eds) Proceedings of national Fusarium head blight forum. East Lansing. Michigan State University, Milwaukee, WI, pp 25-26

Fakhfakh M, Yahyaoui A, Rezgui S et al (2011) Inheritances of Fusarium head blight resistance in a cross involving local and exotic durum wheat cultivars. Crop Sci 51:2517-2524

Garvin D, Stack R, Hansen J (2009) Quantitative trait locus mapping of increased Fusarium head blight susceptibility associated with a wild emmer wheat chromosome. Phytopathology 99:447-452

Ghavami F, Elias E, Mamidi S et al (2011) Mixed model association mapping for Fusarium head blight resistance in Tunisian-derived durum wheat populations. G3 1:209-218

Gladysz C, Lemmens M, Steiner B, Buerstmayr H (2007) Evaluation and genetic mapping of resistance to Fusarium head blight in Triticum dicoccoides. Isr J Plant Sci 55:263-266

Huhn M, Elias E, Ghavami F et al (2012) Tetraploid Tunisian wheat germplasm as a new source of Fusarium head blight resistance. Crop Sci 52:136-145

Kumar S, Stack R, Friesen T, Faris J (2007) Identification of a novel Fusarium head blight resistance quantitative trait locus on chromosome $7 \mathrm{~A}$ in tetraploid wheat. Phytopathology 97:592-597

Oliver R, Stack R, Miller J, Cai X (2007) Reaction of wild emmer wheat accessions to Fusarium head blight. Crop Sci 47:893-899

Oliver R, Cai X, Friesen T, Halley S (2008) Evaluation of Fusarium head blight resistance in tetraploid wheat (L.). Crop Sci 48:213-222 
Otto C, Kianian S, Elias E et al (2002) Genetic dissection of a major Fusarium head blight QTL in tetraploid wheat. Plant Mol Biol 48:625-632

Pestka J (2010) Deoxynivalenol: mechanisms of action, human exposure, and toxicological relevance. Arch Toxicol 84:663-679

Ruan YF, Comeau A, Langevin F, Hucl P, Clarke JM, Brule-Babel A, Pozniak CJ (2012) Identification of novel QTL for resistance to Fusarium head blight in a tetraploid wheat population. Genome 55:853-864

Somers D, Fedak G, Clarke J, Cao W (2006) Mapping of FHB resistance QTLs in tetraploid wheat. Genome 49:1586-1593

Stack R, Elias E, Mitchell Fetch J et al (2002) Fusarium head blight reaction of Langdon durumTriticum dicoccoides chromosome substitution lines. Crop Sci 42:637-642

Talas F, Longin F, Miedaner T (2011) Sources of resistance to Fusarium head blight within Syrian durum wheat landraces. Plant Breed 130:384-400

Taylor R, Koo W (2012) 2012 Outlook of the U.S. and World wheat industries, 2012-2021. Agribus Appl Econ Rep 133393:1-32

Xu X, Nicholson P (2009) Community ecology of fungal pathogens causing wheat head blight. Annu Rev Phytopathol 47:83-103 